\title{
SIRT1 and FOXO Mediate Contractile Differentiation of Vascular Smooth Muscle Cells under Cyclic Stretch
}

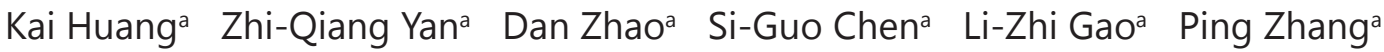 \\ Bao-Rong Shen ${ }^{a}$ Hai-Chao Han ${ }^{a, b}$ Ying-Xin Q $i^{a}$ Zong-Lai Jiang ${ }^{a}$ \\ aInstitute of Mechanobiology \& Medical Engineering, School of Life Sciences \& Biotechnology, \\ Shanghai Jiao Tong University, Shanghai, China; 'Department of Mechanical Engineering, \\ The University of Texas, San Antonio, USA
}

\section{Key Words}

Vascular smooth muscle cells • Cyclic stretch • Differentiation • SIRT1 • FOXO

\begin{abstract}
Background/Aims: Physiological mechanical stretch in vivo helps to maintain the quiescent contractile differentiation of vascular smooth muscle cells (VSMCs), but the underlying mechanisms are still unclear. Here, we investigated the effects of SIRT1 in VSMC differentiation in response to mechanical cyclic stretch. Methods and Results: Rat VSMCs were subjected to $10 \%-1.25 \mathrm{~Hz}$-cyclic stretch in vitro using a FX-4000T system. The data indicated that the expression of contractile markers, including $\alpha$-actin, calponin and $\mathrm{SM} 22 \alpha$, was significantly enhanced in VSMCs that were subjected to cyclic stretch compared to the static controls. The expression of SIRT1 and FOXO3a was increased by the stretch, but the expression of FOXO4 was decreased. Decreasing SIRT1 by siRNA transfection attenuated the stretch-induced expression of contractile VSMC markers and FOXO3a. Furthermore, increasing SIRT1 by either treatment with activator resveratrol or transfection with a plasmid to induce overexpression increased the expression of $\mathrm{FOXO3a}$ and contractile markers, and decreased the expression of FOXO4 in VSMCs. Similar trends were observed in VSMCs of SIRT1 (+/-) knockout mice. The overexpression of FOXO3a promoted the expression of contractile markers in VSMCs, while the overexpression of FOXO4 demonstrated the opposite effect. Conclusion: Our results indicated that physiological cyclic stretch promotes the contractile differentiation of VSMCs via the SIRT1/FOXO pathways and thus contributes to maintaining vascular homeostasis.
\end{abstract}

Copyright $\odot 2015$ S. Karger AG, Basel

\section{Introduction}

Vascular smooth muscle cells (VSMCs) in the media of arterial wall are exposed to mechanical cyclic stretch in vivo, which is caused by lumen pressure [1]. It has been shown that physiological cyclic stretch inhibits VSMC migration, proliferation, and apoptosis and maintains the contractile differentiation of VSMCs, which contributes to vascular homeostasis 


\section{Cellular Physiology Cell Physiol Biochem 2015;37:1817-1829 \begin{tabular}{l|l|l} 
DOI: 10.1159/000438544 & (C) 2015 S. Karger AG, Basel
\end{tabular} \begin{tabular}{l|l|} 
and Biochemistry Published online: November 13, 2015 & www.karger.com/cpb \\
\cline { 1 - 2 }
\end{tabular} \\ Huang et al.: SIRT1 Modulates Stretch Induced VSMC Differentiation}

[2]. However, the underlying mechanism/signal transduction pathways remain unclear.

VSMCs changes from a contractile state to a synthetic state, which plays important roles in vascular remodeling during hypertension and atherosclerosis [3, 4]. In mature vessels, VSMCs mostly have a differentiated contractile state with highly expressed contractile markers, including smooth muscle myosin heavy chain (SM-MHC), SM $\alpha$-actin, SM22 $\alpha$, calponin, and smoothelin, which are associated with the contractile function of VSMCs [5]. VSMCs with differentiated contractile state have low proliferation ratio and migration ability [6]. However, in response to pathological stimulus, such as injury, hypertension and atherosclerosis, VSMCs de-differentiated from a contractile to a synthetic state, which plays a critical role in arterial remodeling [7].

It has been shown that various intracellular signaling pathways are involved in stretchinduced VSMC functions. For example, Rho family GTPases, mitogen-activated protein kinases (MAPKs) and PI3K/Akt initiate the differentiation, migration, and proliferation of VSMCs $[8,9]$. However, the molecular mechanism by which mechanical cyclic stretch modulates differentiation of VSMCs remains to be further elucidated.

SIRT1 is an important $\mathrm{NAD}^{+}$-dependent class III histone deacetylase, and it has been proved to play protective roles in vascular homeostasis [10]. SIRT1 modulates the differentiated response of VSMCs during development and following injury [11, 12]. Pre-Bcell colony-enhancing factor (PBEF) regulates SIRT1 activity by increasing intracellular NAD ${ }^{+}$ via the salvage pathway and promotes VSMC differentiation into a contractile state [13]. In addition, SIRT1 appears to control the cellular responses by regulating the expression and activity of the Forkhead transcription factor (FOXO) family, such as FOXO3a and FOXO4 [14, 15], which have been widely reported to be important molecules in modulating the functions of VSMCs.

Yang et al. reported that FOXO3a regulates the gene transcription of myocardin, which participates in VSMC phenotypic modulation [16]. FOXO3a and its downstream gene, apoptotic protease activating factor 1, play important roles in VSMC survival during vessel remodeling and atherogenesis [17]. FOXO4 and mTORC1 are involved in adiponectininduced VSMC differentiation [18].

Although these studies have revealed that SIRT1, FOXO3a and FOXO4 play important roles in the functional regulation of VSMCs, the effects of SIRT1, FOXO3a and FOXO4 on the differentiation of VSMCs, especially in responsive to mechanical stretch, has not yet been clarified. The objective of this study was to determine whether the SIRT1/FOXO pathway is involved in the cyclic stretch-induced differentiation of VSMCs and to detect the molecular mechanisms that are involved in this process. These results elucidate the biomechanical mechanism that underlies vascular homeostasis and vascular remodeling.

\section{Materials and Methods}

Sirt-1 knockout mice

The animal care and experimental protocols conformed to the Animal Management Rules of China (Documentation 55, 2001, Ministry of Health, China), and the study was approved by the Animal Research Committee of Shanghai Jiao Tong University.

Because Sirt1 homozygous knockout(Sirt1 ${ }^{-/}$) mice have a low perinatal survival rate, Sirt1 heterozygous knockout (Sirt1 ${ }^{+-}$) mice, provided as a gift from Michael W. McBurney at the Center for Cancer Therapeutics, Ottawa Hospital Research Institute, Canada, were used in our studies [19, 20]. Mice were maintained under a specific pathogen-free condition in the vivarium facility of Shanghai Jiao Tong University with a 12 hours light/dark cycle (lights were turned on at 6:00 am). The offspring were genotyped from DNA obtained by a tail clip at weaning $[19,20]$. Male Sirt1 ${ }^{+/-}$mice, 18-20 g in weight, were used for further analysis.

Cell culture

VSMCs were harvested from rat thoracic aorta by an explanted technique [21]. Briefly, the media layer of thoracic aorta was isolated surgically and minced into small pieces that were plated onto $25 \mathrm{~cm}^{2}$ culture 


\section{Cellular Physiology Cell Physiol Biochem 2015;37:1817-1829

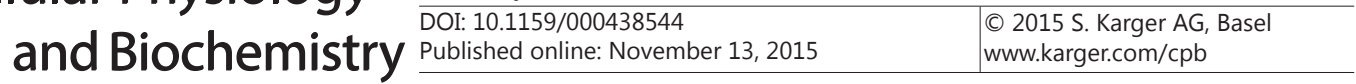 \\ Huang et al:: SIRT1 Modulates Stretch Induced VSMC Differentiation}

flasks in Dulbecco's modified Eagle's medium (DMEM, Gibco) containing 10\% heat-inactivated fetal bovine serum (FBS, Gibco), $100 \mathrm{U} / \mathrm{ml}$ penicillin and $100 \mu \mathrm{g} / \mathrm{ml}$ streptomycin, and incubated at $37^{\circ} \mathrm{C}$ with humidified $5 \% \mathrm{CO}_{2}$. VSMCs displayed the typical spindle-shaped morphology and "hill-and-valley" pattern of growth, and they were characterized by immunohistochemical staining for smooth muscle-specific $\alpha$-actin (Sigma). Cells between passages 4-8 were used.

\section{Application of cyclic stretch}

Rat VSMCs were seeded on type I collagen-coated [21] flexible silicone membrane (Flexercell International, USA $)$ at an initial density of $3 \times 10^{5}$ cells per well $\left(9.32 \mathrm{~cm}^{2}\right)$. After seeding for 24 hours, the cells were starved with DMEM (0\% FBS) for 24 hours to arrest growth and synchronize the cells. VSMCs were then subjected to cyclic stretch that was produced by a computer-controlled vacuum (FX-4000T Strain Unit, Flexercell International, USA) as previously described [21]. The following mechanical parameters were applied: stretch magnitude of $10 \%$, frequency of $1.25 \mathrm{~Hz}$, and duration of 24 hours. VSMCs cultured under the same conditions, but no mechanical stretch applied was used as the static control.

\section{Immunofluorescence staining}

After cyclic stretch application, the attached VSMCs were fixed in 4\% paraformaldehyde at room temperature for 20 minutes, permeabilized in $0.5 \%$ Triton X-100 on ice for 5 minutes, and blocked in 1\% BSA at room temperature for 30 minutes. Then cells were incubated with SIRT1 antibody (1:100, Abcam) at $4^{\circ} \mathrm{C}$ over night. All samples were washed with PBS for 30 minutes and then incubated with an Alexa Fluor 488-conjugated secondary antibody (Cell Signaling Technology, 1:1000) and DAPI for 1 hour at room temperature. The samples were examined with a laser scanning confocal microscope (Olympus, LV1000).

\section{Resveratrol treatment}

Rat VSMCs were seeded at a density of $2.0 \times 10^{5}$ cells per well in six-well plates and grown in DMEM with $10 \%$ FBS. After seeding for 24 hours, VSMCs were starved with DMEM (0\% FBS) for 24 hours. Resveratrol (Sigma) was added to the DMEM culture medium at a concentration of $1 \mu \mathrm{M}, 10 \mu \mathrm{M}, 25 \mu \mathrm{M}, 50 \mu \mathrm{M}$ and 100 $\mu \mathrm{M}$ for 24 hours. Alcohol, which was the vehicle of resveratrol, was used as the control.

\section{RNA interference}

The mRNA sequence of rat SIRT1 (NM_001107627) was acquired from NCBI GenBank. Small interfering RNAs (siRNAs) targeting rat SIRT1 were designed and synthesized by GenePharma Biological Company (Shanghai, P.R. China). The sequences of siRNA for SIRT1 were 5'-GAU UUA UUA CCA GAA ACA ATT-3' and 5'-UUG UUU CUG GUA AUA AAU CTT-3'. The sequences of NC were 5'-UUC UCC GAA CGU GUC ACG UTT-3' and 5'-ACG UGA CAC GUU CGG AGA ATT-3'. Rat VSMCs were seeded at a density of $2.0 \times 10^{5}$ cells per well in six-well plates and grown in DMEM with 10\% FBS. After seeding for 24 hours, the cells were transfected with $100 \mathrm{nmol}$ siRNA and $5 \mu \mathrm{L}$ Lipofectamine ${ }^{\mathrm{TM}} 2000$ (Invitrogen) for 6 hours according to the manufacturer's instruction. Non-silencing siRNA that does not recognize any known genes that are homologous to rat genes was synthesized as a negative control (NC).

\section{SIRT1 overexpression experiment}

pcDNA3-hSIRT1-FLAG mammalian expression plasmid was a kind gift from Dr. Fuyuki Ishikawa (Kyoto University, Graduate School of Biostudies). FLAG-FOXO4 (Addgene plasmid ID 17549) and pcDNA3 flag FKHRL1 AAA (Addgene plasmid ID 10709) plasmid were obtained from Addgene USA. Rat VSMCs were seeded at a density of $2.0 \times 10^{5}$ cells per well in six-well plates, and they were transfected with X-tremeGENE HP DNA Transfection Reagent (Roche) according to the manufacturer's instructions. The transfection efficiency was confirmed by western blot. pcDNA3.1 empty vector was used as the control.

\section{Western blotting}

Mouse thoracic aortas were lysed with homogenizers, and cultured rat VSMCs were lysed for 5 minutes in lysis buffer at $4^{\circ} \mathrm{C}$. Respective nuclear and cytoplasmic proteins from VSMCs were extracted by using a Nuclear and Cytoplasmic Protein Extraction Kit (Beyotime Institute of Biotechnology, China). Protein concentration was determined by an Enhanced BCA Protein Assay Kit (Beyotime Institute of Biotechnology, China). $30 \mu \mathrm{g}$ proteins (per lane) were subjected to electrophoretic separation by $10 \%$ SDSPAGE and transferred to nitrocellulose membranes (Hybond, Amersham). Western blots were performed using antibodies directed against SIRT1 (1:300, Santa Cruz Biotechnology), FOXO4 (1:300, Cell Signaling 


\section{Cellular Physiology Cell Physiol Biochem 2015;37:1817-1829 \begin{tabular}{l|l|l}
\hline DOI: 10.1159/000438544 & (C) 2015 S. Karger AG, Basel
\end{tabular} www.karger.com/cpb

Technologies), FOXO3a (1:500, Cell Signaling Technologies), SM22 $\alpha$ (1:800, Abcam), smooth muscle $\alpha$-actin (1:1000, Sigma), calponin (1:500, Sigma) and GAPDH (1:1000, Santa Cruz Biotechnology). After incubation with alkaline phosphatase-conjugated secondary antibodies (Jackson Immunoresearch), the signals were visualized by nitroblue tetrazolium-bromochloroindolyl phosphate (Bio Basic, Inc.), and quantified using Quantity One software (Bio-Rad).

\section{Ingenuity Pathway Analysis}

The potential diseases and functions of FOXO3a or FOXO4 in cardiovascular system and the possible networks among FOXO3a, FOXO4 and SIRT1 were obtained by Ingenuity Pathway Analysis (IPA) software (http://www.ingenuity.com/products/ipa). The significance values for analyses were calculated by comparing the molecule that participates in a given function or pathway relative to the total number of occurrences in all functional/pathway annotations stored in the Ingenuity Pathways Knowledge Database Literature.

\section{Statistical analysis}

Each experiment was performed at least in triplicate, and all values are expressed as mean \pm SD. Oneway ANOVA was used to compare the difference between the two groups followed by Fisher's $t$-test for multiple comparisons. Values of $P<0.05$ were accepted as statistically significant.

\section{Results}

Mechanical stretch modulated expression of SIRT1 in VSMCs

Compared to the static control, $10 \%$ cyclic stretch significantly increased the expression level of SIRT1 in VSMCs (Fig. 1A). The expression of SIRT1 in nucleus and cytoplasm are both significantly increased (Fig. 1B, 1C, 1D), and the increase in the nucleus was more remarkable than that in the cytoplasm (Fig. 1B, 1C).

Fig. 1. Cyclic stretch regulates the expression of SIRT1 in VSMCs. A $10 \%-1.25 \mathrm{~Hz}$ cyclic stretch remarkably increased the expression level of SIRT1 in VSMCs (A). The increase of SIRT1 is detected in both nucleus (B) and cytoplasm (C). GAPDH is used as a housekeeping control for total protein and cytoplasmic protein; TBP is used as a housekeeping control for nuclear protein. Values are shown as Mean \pm S.D. $* P<$ 0.05 , ** $P<0.01$ (n=5). Immunofluorescence staining reveals that compared with static control, $10 \%-1.25 \mathrm{~Hz}$ cyclic stretch notably increases the expression of SIRT1 in nucleus and cytoplasm of VSMCs $($ Bar $=50 \mu \mathrm{m})(\mathrm{D})$.

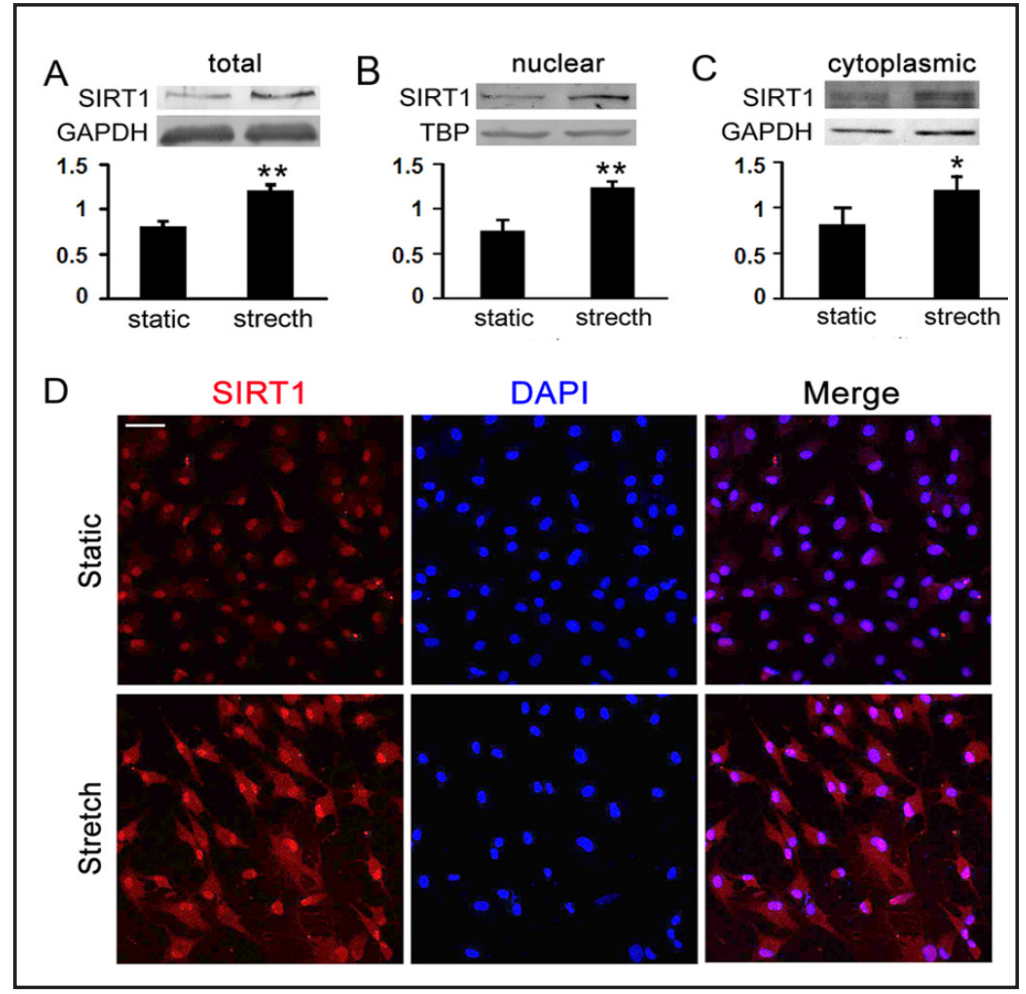




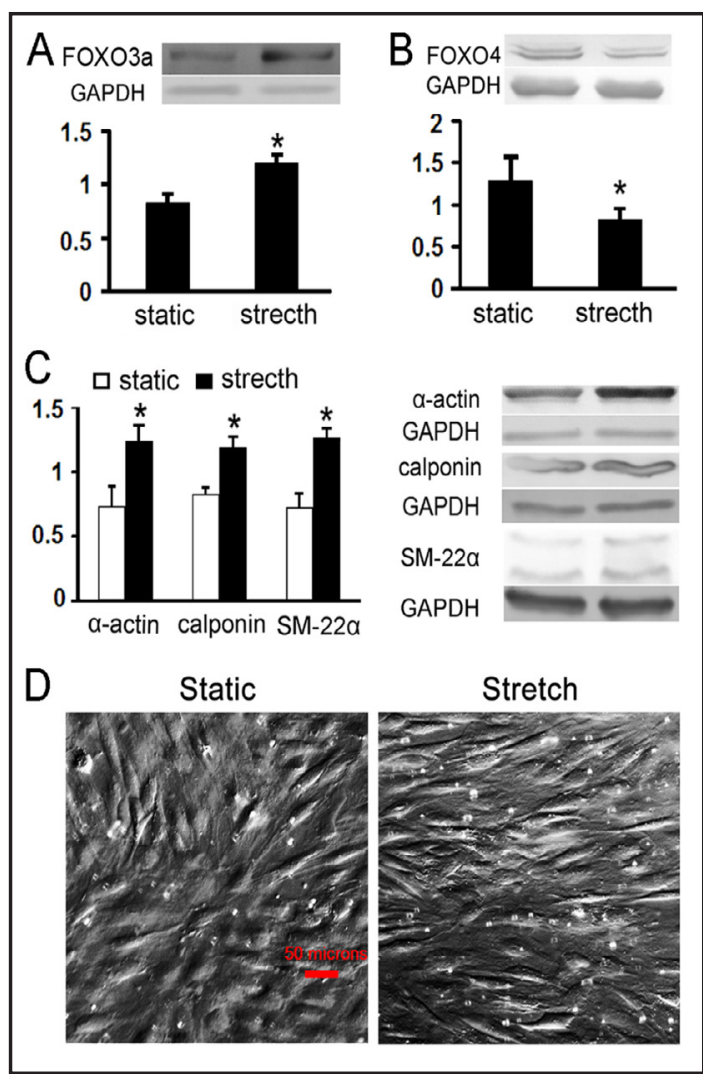

Fig. 2. Cyclic stretch regulates the expression of FOXO3a and FOXO4 as well as the differentiation of VSMCs. A $10 \%-1.25 \mathrm{~Hz}$ cyclic stretch notably increases the expression level of FOXO3a (A), decreases the expression level of FOXO4 (B), and induces the expression of differentiated markers in VSMCs, including $\alpha$-actin, SM22 $\alpha$ and calponin (C) compared to the static control. GAPDH is used as a housekeeping control. Values are shown as Mean \pm S.D. ${ }^{*} P<0.05$, ** $P<0.01(\mathrm{n}=5)$. Microscopic photograph revealed that cyclic-stretch-application elongated VSMCs $($ Bar $=50 \mu \mathrm{m})(\mathrm{D})$.

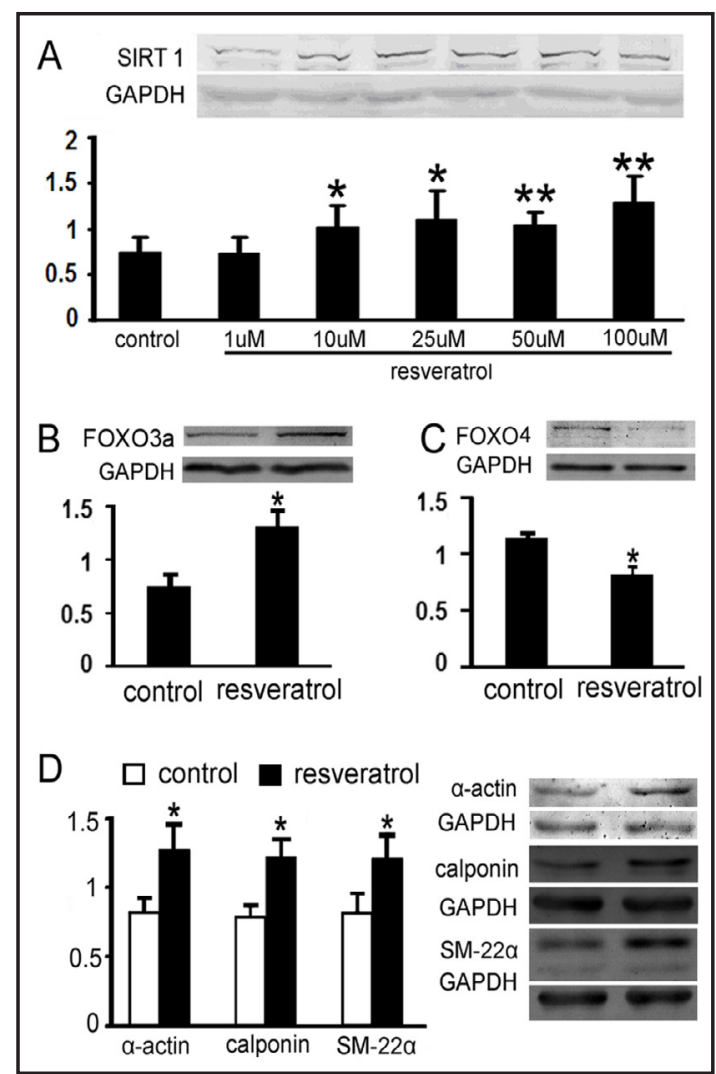

Fig. 3. Elevation of SIRT1 by resveratrol regulates the expression of FOXO3a and FOXO4 and differentiated markers of VSMCs. $1 \mu \mathrm{M}$ resveratrol treatment has no significant effect on the expression of SIRT1, while $10 \mu \mathrm{M}, 25 \mu \mathrm{M}, 50 \mu \mathrm{M}$ and $100 \mu \mathrm{M}$ resveratrol all significantly increases the expression of SIRT1 (A). Resveratrol treatment ( $25 \mu \mathrm{M}$ for 24 hours) significantly increases the expression of FOXO3a (B) and contractile markers, $\alpha$-actin, SM22 $\alpha$, and calponin (D), but decreases the expression of FOXO4 (C). GAPDH is used as a housekeeping control. Values are shown as Mean $\pm S . D .{ }^{*} P<0.05 v s$ the control $(\mathrm{n}=4)$.

Mechanical stretch modulated expression of FOXO3a, FOXO4 and contractile differentiated markers in VSMCs

Our results revealed that a $10 \%$ mechanical stretch significantly increased the expression level of the transcription factor FOXO3a but decreased the expression level of FOXO4 (Fig. $2 \mathrm{~A}, 2 \mathrm{~B})$. The expression levels of contractile VSMC markers, including $\alpha$-actin, SM22 $\alpha$ and calponin were all increased by the application of mechanical stretch (Fig. 2C) compared to the static control. Microscopic photograph revealed that after cyclic-stretch application, VSMCs were elongated and almost perpendicular aligned to the stretch direction (Fig. 2D).

These in vitro results revealed that a physiological stretch that is $10 \%$ cyclic stretch [21], maintains the VSMC contractile differentiation.

Resveratrol modulated expression of FOXO3a, FOXO4 and contractile differentiated markers in VSMCS

To further study the role of SIRT1, FOXO3a and FOXO4 in response to mechanical stretch, resveratrol, an activator of SIRT1 [22] was used to stimulate SIRT1 in VSMCs under 


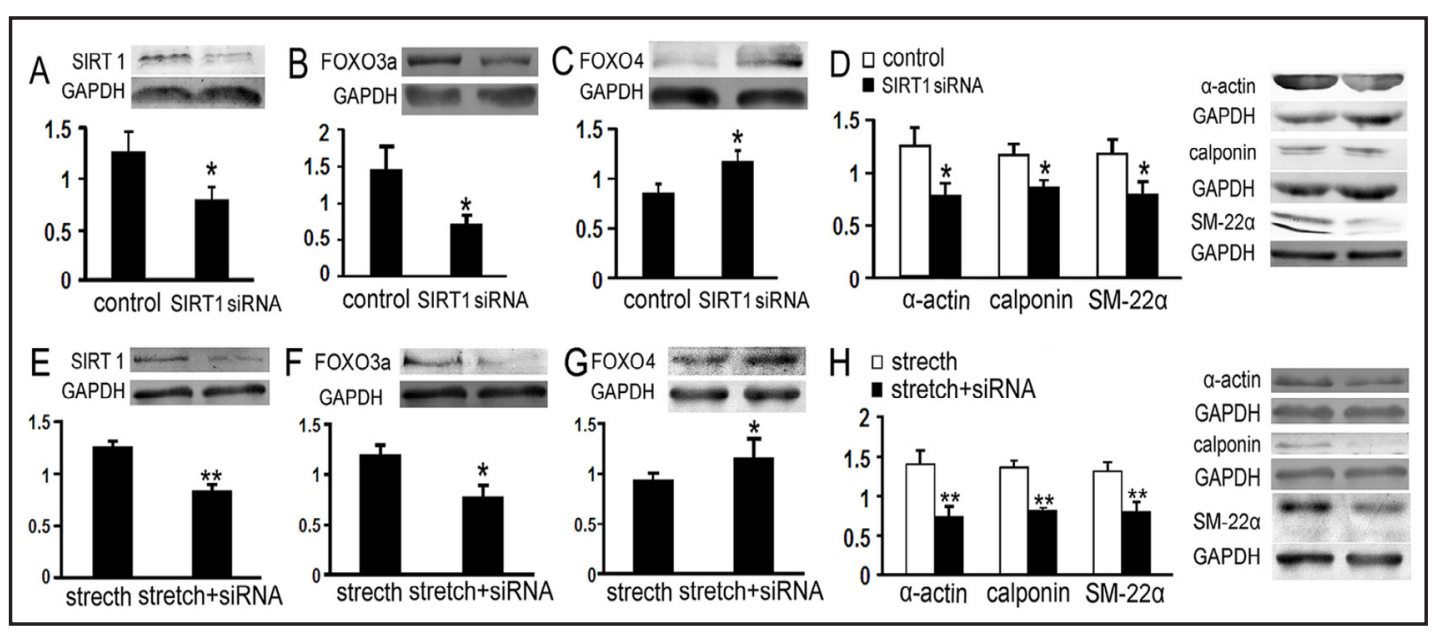

Fig. 4. Effects of SIRT1 target siRNA on the expression of FOXO3a, FOXO4 and differentiated markers of VSMCs under static and cyclic stretch. Under static condition, target siRNA significantly represses the expression of SIRT1 (A), FOXO3a (B) and differentiated markers, $\alpha$-actin, SM22 $\alpha$, and calponin (D), but increases the expression of FOXO4 (C). Under 10\%-1.25 Hz-cyclic-stretch application, SIRT1 target siRNA decreases the expression of SIRT1 (E), FOXO3a (F), as well as the differentiated markers of VSMCs, including $\alpha$-actin, SM22 $\alpha$ and calponin $(H)$, but increased the expression of FOXO4 (G). GAPDH is used as a housekeeping control. Values are shown as Mean \pm S.D. ${ }^{*} P<0.05$, ** $P<0.01$ vs the negative control (NC) $(\mathrm{n}=5)$.

static conditions. The results showed that VSMCs treated with resveratrol $(1 \mu \mathrm{M})$ did not have a significant effect on the expression of SIRT1, whereas $10 \mu \mathrm{M}, 25 \mu \mathrm{M}, 50 \mu \mathrm{M}$ and 100 $\mu \mathrm{M}$ Resveratrol all significantly increased the expression of SIRT1 (Fig. 3A). Furthermore, resveratrol $(25 \mu \mathrm{M})$ treatment significantly increased the expression of FOXO3a (Fig. 3B), decreased the expression of FOXO4 (Fig.3C), and increased contractile VSMC markers (Fig. 3D), indicating that resveratrol treatment increased the expression of FOXO3a and contractile protein markers but decreased the expression of FOXO4.

SIRT1 siRNA or overexpression modulated expression of FOXO3a, FOXO4 and contractile differentiated markers in VSMCs

SIRT1 target siRNA transfection in VSMCs decreased the expression of SIRT1 (Fig. 4A), which repressed FOXO3a expression (Fig. 4B) but elevated FOXO4 expression (Fig. 4C). The expression of contractile markers was decreased by SIRT1 target siRNA transfection (Fig. 4D).

Furthermore, when VSMCs were transfected with SIRT1 siRNA and then subjected to a 10\% cyclic stretch, SIRT1 siRNA decreased the expression of SIRT1 (Fig. 4E) and repressed the expression of FOXO3a (Fig. 4F), whereas the expression of FOXO4 was increased in this condition (Fig. 4G). The expression of contractile markers was decreased in this group (Fig. 4H).

Transfection with the SIRT1 overexpression plasmid revealed that the up regulated SIRT1 (Fig. 5A) increased the expression of FOXO3a (Fig. 5B) and contractile VSMC markers, i.e., $\alpha$-actin, SM22 $\alpha$ and calponin (Fig. 5D), but decreased the expression of FOXO4 (Fig. 5C).

These results suggest that SIRT1, whose expression was changed by mechanical stretch, has opposite effects on the expression of FOXO3a and FOXO4, which could subsequently regulate VSMC differentiation.

FOXO3a or FOXO4 overexpression modulated expression of contractile markers in VSMCS

To further detect the changed expression of FOXO3a and FOXO4 upon VSMC differentiation, FOXO3a or FOXO4 overexpression plasmids were transfected into VSMCs 


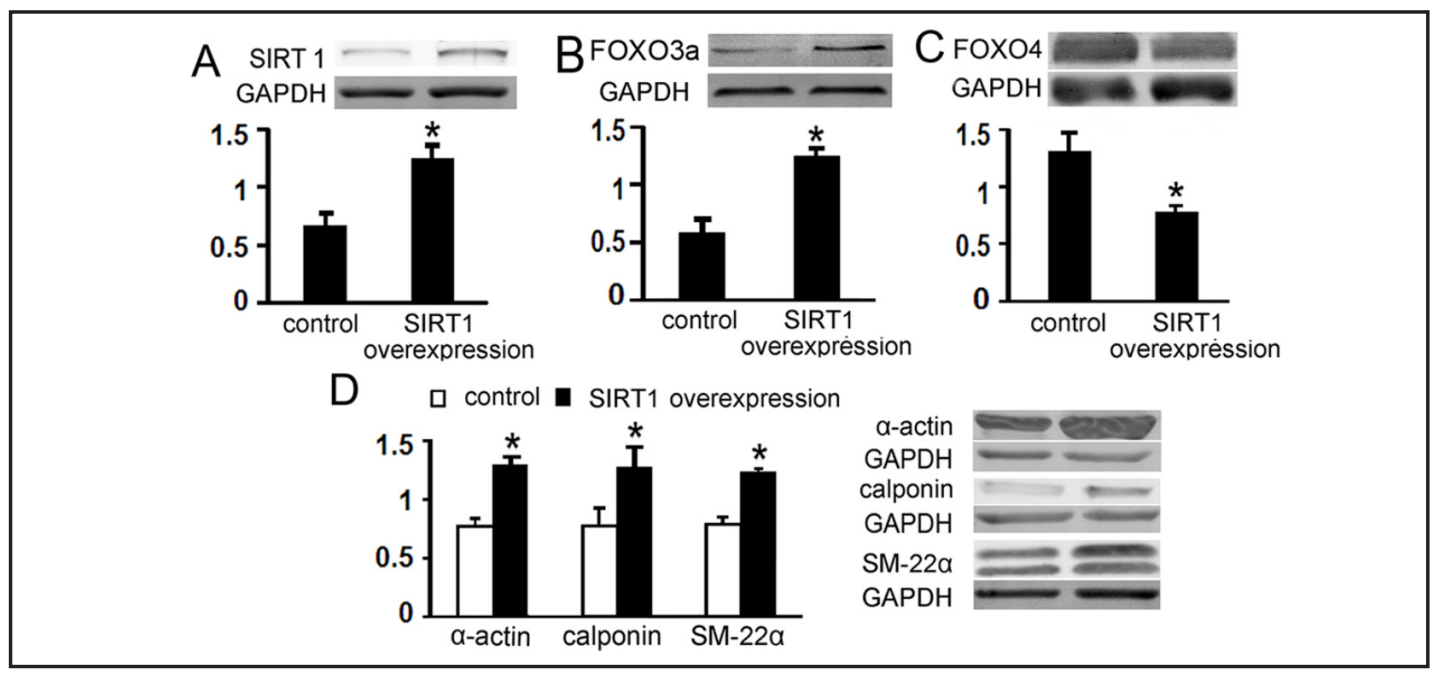

Fig. 5. Effects of SIRT1 overexpression on the expression of FOXO3a and FOXO4 and the differentiation of VSMCs. Overexpression of SIRT1 by transfection with pcDNA3.1-SIRT1-Flag plasmid (A) increases the expression of FOXO3a (B), but decreases FOXO4 (C). The expression of VSMC contractile state markers, including, $\alpha$-actin, SM22 $\alpha$, and calponin (D) is significantly increased. GAPDH is used as a housekeeping control. Values are shown as Mean \pm S.D. ${ }^{*} P<0.05$ vs the pcDNA3.1 control $(\mathrm{n}=3)$.

Fig. 6. Effects of FOXO3a or FOXO4 overexpression on the differentiation of VSMCs. Overexpression of FOXO3a by transfection with pcDNA3.1-FOXO3a-Flag plasmid $(A)$ increases the expression of VSMC contractile markers, $\alpha$-actin, SM $22 \alpha$ and calponin (C). Overexpression of FOXO4 by transfection with pcDNA3.1-FOXO4-Flag plasmid (B) decreases the expression of VSMC contractile markers $\alpha$-actin, SM22 $\alpha$ and calponin (D). GAPDH is used as a housekeeping control. Values are shown as Mean \pm S.D. $* P<0.05$ vs the pcDNA3.1 control $(\mathrm{n}=4)$.

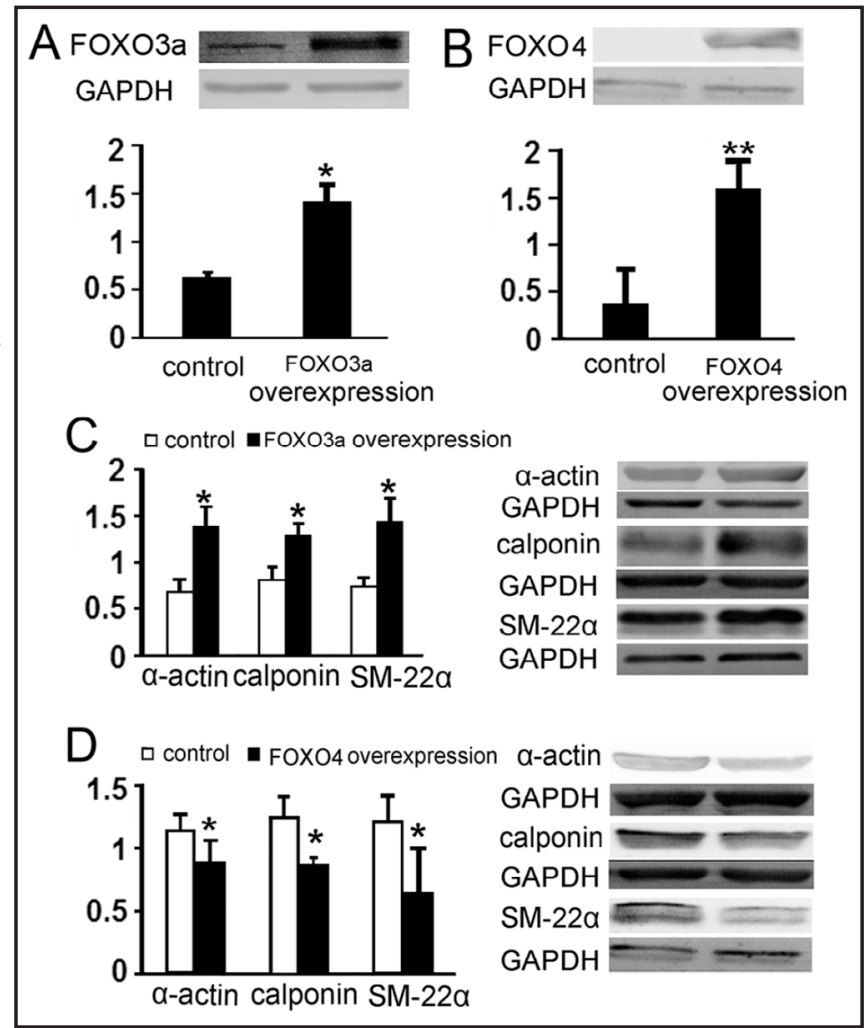

under static condition. The data indicated that overexpressed FOX03a (Fig. 6A) increased the expression of $\alpha$-actin, SM22 $\alpha$ and calponin (Fig. 6C), while overexpressed FOXO4 (Fig. 6B) decreased the expression of $\alpha$-actin, SM22 $\alpha$ and calponin (Fig. 6D). These results suggested that the altered expression of FOXO3a and FOXO4 plays different roles in VSMC differentiation.

\section{KARGER}




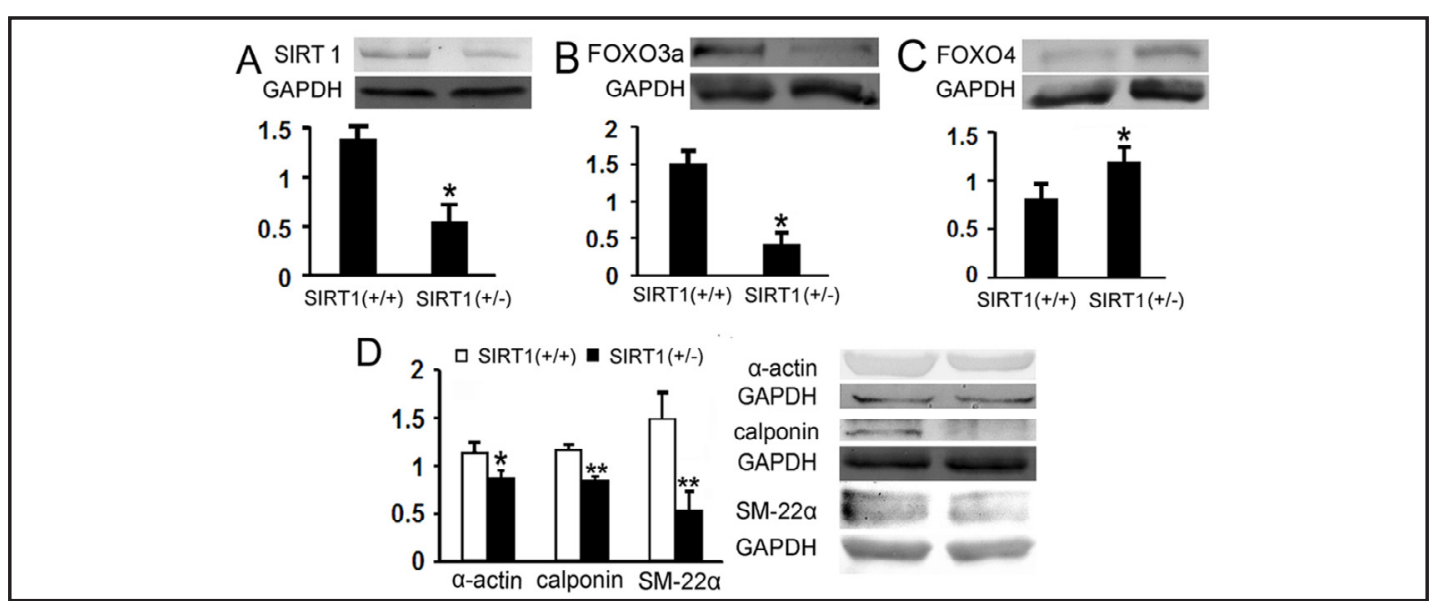

Fig. 7. Expression of SIRT1, FOXO3a and FOXO4 and differentiated markers of VSMCs in thoracic aorta of SIRT1 ${ }^{+/-}$mice. Compared to wild type SIRT ${ }^{+/+}$mice, the expression of SIRT1 (A) FOXO3a (B), and contractile markers, $\alpha$-actin, SM22 $\alpha$ and calponin (D) is repressed, but the expression of FOXO4 is significantly increased (C) in SIRT1 ${ }^{+/-}$mice. GAPDH is used as a housekeeping control. Values are shown as Mean \pm S.D. ${ }^{*} P<0.05$ vs the wild type mice (SIRT $\left.{ }^{+/+}\right),{ }^{* *} P<0.01$ vs the wild type mice $\left(\mathrm{SIRT}^{+/+}\right)(\mathrm{n}=5)$.

\section{SIRT1 knockout in vivo decreased differentiated markers in mice thoracic aorta}

Because Sirt1 homozygous knockout mice have a low perinatal survival rate $[19,20]$, Sirt1 heterozygous knockout (SIRT1 ${ }^{+/-}$) mice were used to decrease the expression of SIRT1 and to further validate our findings in vivo. Compared with the wild type (SIRT ${ }^{+/+}$) mice (male), the expression of SIRT1 in thoracic aorta from SIRT1 ${ }^{+/-}$mice (male) was repressed (Fig. 7A). The expression of FOXO3a was repressed (Fig. 7B), but the expression of FOXO4 was significantly increased in VSMCs (Fig. 7C). The expression of contractile VSMC markers was also repressed (Fig. 7D) compared with that in the wild type mice.

\section{Discussion}

Our results demonstrated that cyclic stretch increased the expression of SIRT1, FOXO3a and contractile VSMC markers, but decreased the expression of FOXO4. These increases were repressed by decreasing SIRT1 with SIRT1 siRNA transfection, whereas increasing SIRT1 by resveratrol or transfection of the SIRT1-overexpression plasmid increased the expression of the contractile markers. The results demonstrated that the cyclic stretch modulated VSMC differentiation was affected by the SIRT1-FOXO pathway (Fig. 8A).

Blood vessels are subjected to mechanical stretch due to the pulsatile nature of blood flow, and mechanical stretch profoundly influences the orientation, morphology, migration, proliferation, apoptosis and phenotype of VSMCs [23, 24]. Pathologically increased cyclic stretch contributes to vascular remodeling during aging and hypertension $[25,26]$, whereas the physiological level of cyclic stretch is essential for maintaining the vascular wall structure and inhibiting the growth factor-stimulated proliferation of VSMCs [27]. Our present results showed that physiological cyclic stretch (10\%-1.25 Hz) significantly increased the expression of VSMC contractile markers. The similar physiological responses of VSMCs are also revealed by culture of whole vascular tissue $[26,28]$. Therefore, physiological cyclic stretch is a protective stimulus to maintain the contractile differentiation of VSMCs and to maintain vascular homeostasis.

SIRT1, as an important class III histone deacetylase, participates in many cell functions, i.e., senescence, proliferation, differentiation, and apoptosis $[2,29,30]$. It has been shown that mammalian SIRT1 is involved in chromatin remodeling, gene silencing, DNA damage response [31], and caloric restriction [32]. Activation of SIRT1 induced by caloric restriction 
Fig. 8. The relationships among SIRT1 and FOXOs. (A) Schematic drawing of outlines the possible signaling pathway of SIRT1- FOXOs. 10\% cyclic stretch loading on the VSMCs activates the expression of SIRT1, affects expression of FO$\mathrm{XOs}$, and then induces VSMC differentiation. $\longrightarrow$ indicates promotion; $\longrightarrow$ indicates inhibition. (B) The potential relationships among SIRT1, FOX03a and FOXO4 in mammal cells, revealed by IPA bioinformatics software. - (without arrowheads) refers to a binding interaction. ....... (dotted line) indicates an inferred or indirect interaction. $\bigcirc$ indicates transcription regulator, $\diamond$ indicates enzyme, 0 indicates group or complex, $\nabla$ indicates kinase, $\bigcirc$ indicates other.

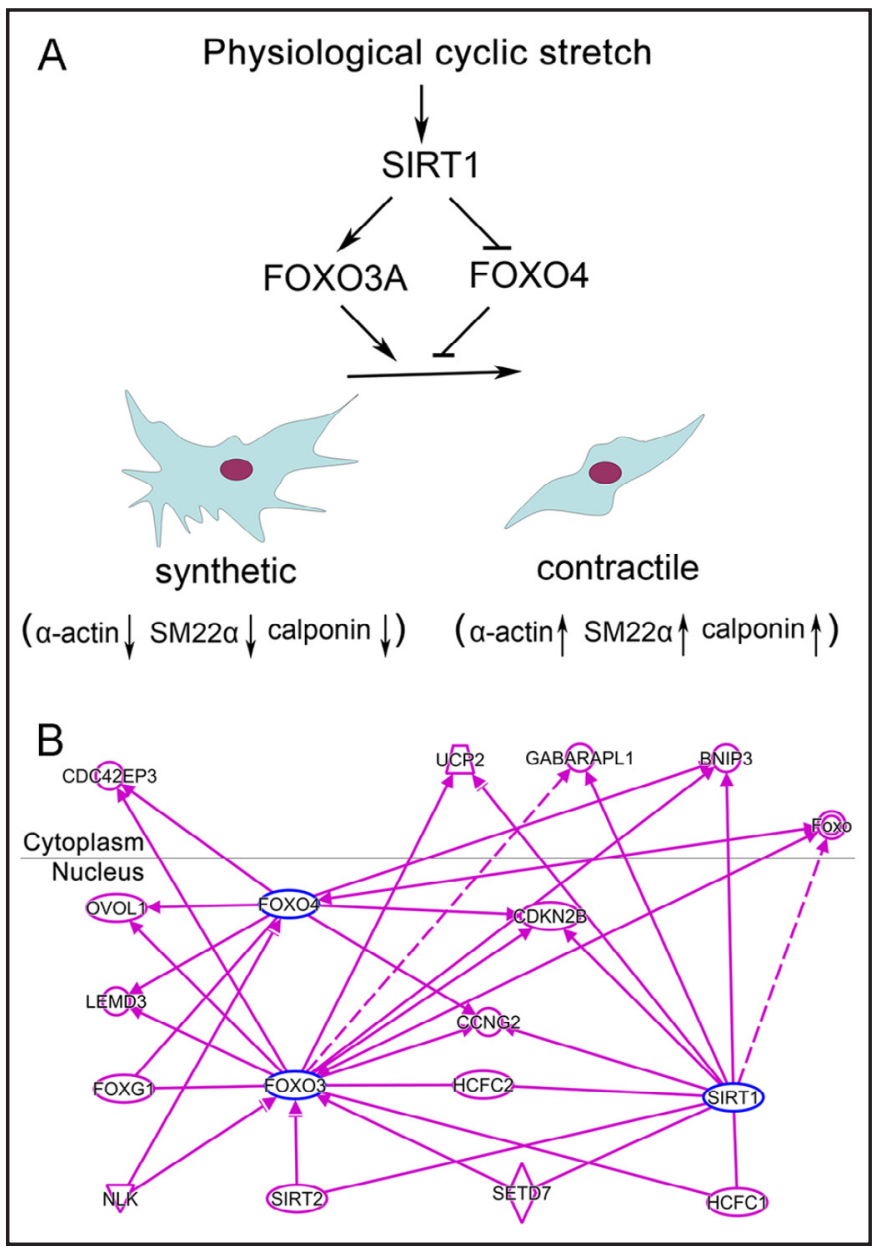

significantly attenuates age-related vascular oxidative stress and inflammation, and improves endothelial homeostasis [33, 34]. Moderate overexpression of SIRT1 in the heart of transgenic mice attenuated age-dependent increases in cardiac hypertrophy, apoptosis/ fibrosis, cardiac dysfunction, and the expression of senescence markers [35]. A recent study revealed that in VSMCs, SIRT1 protects against oxidized low-density lipoprotein-induced DNA damage and senescence and inhibits medial degeneration and atherosclerosis [36]. The results from these studies suggest a protective effect of SIRT1 on the cardiovascular system.

The new findings of our present study revealed that SIRT1 is a crucial mechanoresponsive molecule. Kim et al. recently revealed that the expression of SIRT1 is regulated by microRNA-34a [37], which had been proved to be an important mechano-sensitive molecule in our previous work [38]. Interestingly, our unpublished data revealed that physiological cyclic stretch decreases the expression of microRNA-34a in VSMCs. Whether the decreased expression of microRNA-34a can target on SIRT1 and regulate its expression in VSMCs will be examined in the future.

SIRT1 has been proved to play critical roles in vascular homeostasis and remodeling [22, 39]. Here, using SIRT1 activator-resveratrol, SIRT1 overexpression plasmid, specific siRNA transfection, and knockout mice, we revealed the protective role of SIRT1 in maintaining the normal contractile differentiation of VSMCs. Furthermore, physiological cyclic stretch increased SIRT1 expression, which subsequently modulated the expression of FOXO3a and FOXO4 and then the differentiation of VSMCs.

It has been revealed that SIRT1 deacetylates FOXOs, thus attenuating cell apoptosis and cell-cycle arrest [40]. Acetylated FOXO3a was deacetylated by SIRT1 in an NAD-dependent manner [40]. Treatment with nicotinamide, a SIRT1 inhibitor, results in the deacetylation of 


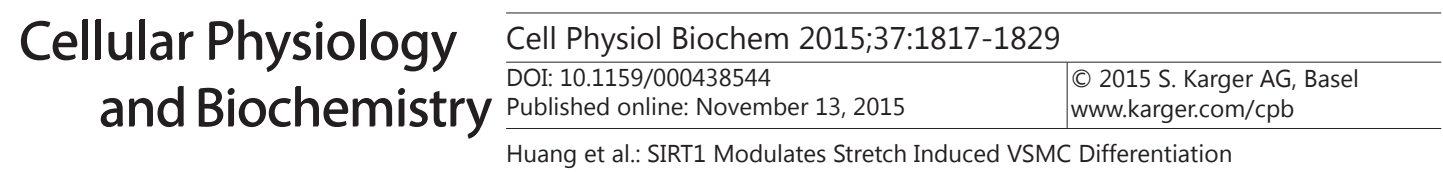

Table 1. The top 10 cellular functions and diseases of cardiovascular system related to FOXO3a analyzed by Ingenuity Pathway Analysis

\begin{tabular}{lll}
\hline Categories & Diseases or Functions Annotation & p-Value \\
\hline Cell Cycle & arrest in mitosis of erythroid cells & $3.40 \mathrm{E}-05$ \\
& $\begin{array}{l}\text { arrest in cell cycle progression of squamous cell } \\
\text { carcinoma cell lines }\end{array}$ & $1.70 \mathrm{E}-04$ \\
& $\begin{array}{l}\text { arrest in G0/G1 phase transition of leukemia cell } \\
\text { lines }\end{array}$ & $3.06 \mathrm{E}-04$ \\
& exit from S phase of colon cancer cell lines & $3.40 \mathrm{E}-04$ \\
& hyperactivation of helper T lymphocytes & $3.40 \mathrm{E}-05$ \\
Cell-To-Cell Signaling and Interaction, Inflammatory & & \\
Response & immune complex-mediated inflammatory arthritis & $6.81 \mathrm{E}-05$ \\
Connective Tissue Disorders, Inflammatory Disease, & & \\
Skeletal and Muscular Disorders & & $1.02 \mathrm{E}-04$ \\
Cell Death and Survival & apoptosis of MCTC mast cell & $1.02 \mathrm{E}-04$ \\
& apoptosis of mucosal mast cells & $1.02 \mathrm{E}-04$ \\
& loss of neuronal progenitor cells & $5.79 \mathrm{E}-04$ \\
& atrophy of myofiber & $9.87 \mathrm{E}-04$ \\
& apoptosis of natural killer cells & $1.02 \mathrm{E}-04$ \\
Endocrine System Disorders, Hematological Disease, & fasting hypoglycemia & \\
Metabolic Disease & & $1.02 \mathrm{E}-04$ \\
Free Radical Scavenging & scavenging of hydrogen peroxide & $4.08 \mathrm{E}-04$ \\
Cardiovascular System Development and Function & hyperproduction of reactive oxygen species & $1.02 \mathrm{E}-04$ \\
& growth of atretic ovarian follicle & $1.36 \mathrm{E}-04$ \\
& arrest in growth of endothelial cells & $6.13 \mathrm{E}-04$ \\
Cellular Compromise, Connective Tissue Disorders, & maturation of blood vessel & damage of red blood cells \\
Organismal Injury and Abnormalities & atrophy of myotube & $1.36 \mathrm{E}-04$ \\
Inflammatory Response & Sjogren's-syndrome like inflammation & $2.72 \mathrm{E}-04$ \\
& memory T cell response & $1.70 \mathrm{E}-04$ \\
Cellular Assembly and Organization, Cellular & inflammation of salivary gland & $4.08 \mathrm{E}-04$ \\
Compromise & disruption of mitochondrial membrane & $7.83 \mathrm{E}-04$ \\
& & $1.70 \mathrm{E}-04$ \\
\hline
\end{tabular}

Table 2. The top 10 cellular functions and diseases of cardiovascular system related to FOXO4 analyzed by Ingenuity Pathway Analysis

\begin{tabular}{|c|c|c|}
\hline Categories & Diseases or Functions Annotation & $\mathrm{p}$-Value \\
\hline $\begin{array}{l}\text { Endocrine System Disorders, Hematological Disease, } \\
\text { Metabolic Disease }\end{array}$ & fasting hypoglycemia & $1.02 \mathrm{E}-04$ \\
\hline Cell Morphology, Connective Tissue Development and & cell flattening of fibroblast cell lines & $2.38 \mathrm{E}-04$ \\
\hline Function & size of fibroblast cell lines & $6.81 \mathrm{E}-04$ \\
\hline \multirow[t]{2}{*}{ Cell Death and Survival, Cellular Development } & $\begin{array}{l}\text { self-renewal of hematopoietic progenitor cells } \\
\text { apoptosis of endothelial cells }\end{array}$ & $1.26 \mathrm{E}-03$ \\
\hline & & $1.19 \mathrm{E}-02$ \\
\hline Cellular Development, Cellular & proliferation of myeloid progenitor cells & $1.33 \mathrm{E}-03$ \\
\hline \multirow{2}{*}{ Proliferation, Tissue Development } & differentiation of smooth muscle cells & $2.45 \mathrm{E}-03$ \\
\hline & proliferation of fibroblasts & $1.89 \mathrm{E}-02$ \\
\hline Nucleic Acid Metabolism & quantity of NAD+ & $1.36 \mathrm{E}-03$ \\
\hline Connective Tissue Development and Function & focus formation of fibroblast cell lines & $1.50 \mathrm{E}-03$ \\
\hline \multirow{2}{*}{ Gene Expression } & transcription of mRNA & $2.04 \mathrm{E}-03$ \\
\hline & transactivation of RNA & $2.94 \mathrm{E}-02$ \\
\hline $\begin{array}{l}\text { Cellular Assembly and Organization, Lipid Metabolism, } \\
\text { Small Molecule Biochemistry }\end{array}$ & accumulation of lipid droplets & $2.49 \mathrm{E}-03$ \\
\hline $\begin{array}{l}\text { Cardiovascular Disease, Hematological Disease, } \\
\text { Metabolic Disease }\end{array}$ & Hypertriglyceridemia & $3.64 \mathrm{E}-03$ \\
\hline Cardiovascular System Development and Function & formation of neointima & $3.64 \mathrm{E}-03$ \\
\hline
\end{tabular}

FOX03 in 293T cells $[41,42]$. SIRT1 can also participate in FOXOs-induced cellular functions via modulating the expression of FOXOs. Our results showed that the expression of FOXO4 was decreased, while the expression of FOXO3a was increased by SIRT1. It has also been reported by other studies that other than deacetylation, the expression of FOXOs is also regulated by SIRT1 in several types of cells $[14,15]$. Recent research demonstrated that host cell factor 1 (HCF-1) participates in the SIRT1-regulated expression of FOXOs in worms [15]. 


\section{Cellular Physiology Cell Physiol Biochem 2015;37:1817-1829 \begin{tabular}{ll|l} 
DOI: 10.1159/000438544 & $\begin{array}{l}\text { O 2015 S. Karger AG, Basel } \\
\text { www.karger.com/cpb }\end{array}$ \\
\hline
\end{tabular} \\ Huang et al.: SIRT1 Modulates Stretch Induced VSMC Differentiation}

FOXOs, as important transcriptional regulators, play important roles in the maintenance of vascular homeostasis and vascular stability [43]. FOXO3a is a negative transcription factor of CYR61 which is crucial for VSMC proliferation and neointimal hyperplasia [44]. Here we revealed a protective role of increased FOXO3a in maintaining the differentiation of VSMCs, but a pathological role of increased FOXO4. Liu et al. also reported that FOXO4 inhibits contractile genes expression in VSMCs [45]. IPA bioinformatics analysis suggested that FOXO3a and FOXO4 participates in various cellular functions in cardiovascular system, including cell cycle, cell death and survival, cellular assembly and organization, cellular growth and proliferation, cellular development, cell morphology, and et al. (Table 1,2). Since contractile differentiated VSMCs shows a less intent to proliferate, migrate, and synthesize extracellular matrix (ECM), which contribute to maintain vascular homeostasis [2], the roles of FOXO3a and FOXO4 on VSMC functions, such as proliferation, migration, apoptosis and synthesize, need further demonstration.

Although our present research suggested that SIRT1 may be the upstream molecule of FOXO3a and FOXO4 in stretch-induced VSMC phenotypic modulation, the underlying mechanism in this process is complex and requires further study. Using IPA bioinformatics software, the potential relationships among SIRT1, FOXO3a and FOXO4 in mammal cells were revealed (Fig. 8B). It suggested that many transcription factors may participate in this regulating network, including cyclin-dependent kinase inhibitor 2B (CDKN2B), cyclin G2 (CCNG2), host cell factor C1 (HCFC1), host cell factor C2 (HCFC2), SIRT2, forkhead box G1 (FOXG1), and ovo-like zinc finger 1 (OVOL1). However, the effects of these transcription factors on the expression of FOX03a and FOXO4 modulated by SIRT1 in VSMCs are still unclear, which need to be demonstrated in the future.

In conclusion, our studies suggest that the up regulation of SIRT1 by physiological cyclic stretch induced contractile differentiation of VSMCs, and the opposite variation of FOXO3a and FOXO4 are involved in this process. These results define a novel role of SIRT1 as an important regulator to maintain vascular homeostasis, and the results also raise the possibility that the activation of SIRT1 could be a useful strategy for treating pathological vascular remodeling in hypertension and other vascular diseases.

\section{Acknowledgments}

This research was supported by grants from the National Natural Science Foundation of China, Nos. 11232010, 10972120, 11229202 and 11172176.

We thank Dr. Fuyuki Ishikawa at the Graduate School of Biostudies, Kyoto University for the kind gift of the pcDNA3-hSIRT1-FLAG plasmid. We thank Dr. Domenico Accili at Addgene for providing the FLAG-FOXO4 plasmid and Dr. William Sellers at Addgene for providing the pcDNA3 flag FKHRL1 AAA. We also thank Michael W. McBurney at the Center for Cancer Therapeutics, Ottawa Hospital Research Institute, Canada for the kind gift of the Sirt1 transgenic mice.

\section{Disclosure Statement}

None declared.

\section{References}

1 Haga JH, Li YS, Chien S: Molecular basis of the effects of mechanical stretch on vascular smooth muscle cells. J Biomech 2007;40:947-960.

2 Han Y, Fukuda N, Ueno T, Endo M, Ikeda K, Xueli Z, Matsumoto T, Soma M, Matsumoto K: Role of complement $3 \mathrm{a}$ in the synthetic phenotype and angiotensin II-production in vascular smooth muscle cells from spontaneously hypertensive rats. Am J Hypertens 2012;25:284-289. 


\section{Cellular Physiology Cell Physiol Biochem 2015;37:1817-1829 \begin{tabular}{l|l} 
and Biochemistry Published online: November 13, 2015 & $\begin{array}{l}\text { DOI: 2015 S. Karger AG, Basel } \\
\text { www.karger.com/cpb }\end{array}$ \\
\hline
\end{tabular} \\ Huang et al.: SIRT1 Modulates Stretch Induced VSMC Differentiation}

3 Qi YX, Qu MJ, Yan ZQ, Zhao D, Jiang XH, Shen BR, Jiang ZL: Cyclic strain modulates migration and proliferation of vascular smooth muscle cells via Rho-GDIalpha, Rac1, and p38 pathway. J Cell Biochem 2010;109:906-914.

4 Stintzing S, Ocker M, Hartner A, Amann K, Barbera L, Neureiter D: Differentiation patterning of vascular smooth muscle cells (VSMC) in atherosclerosis. Virchows Arch 2009;455:171-185.

5 Shanahan CM, Weissberg PL: Smooth muscle cell heterogeneity: patterns of gene expression in vascular smooth muscle cells in vitro and in vivo. Arterioscler Thromb Vasc Biol 1998;18:333-338.

6 Carrillo-Sepulveda MA, Matsumoto T: Phenotypic modulation of mesenteric vascular smooth muscle cells from type 2 diabetic rats is associated with decreased caveolin- 1 expression. Cell Physiol Biochem 2014;34,1497-1506.

7 Lagna G, Ku MM, Nguyen PH, Neuman NA, Davis BN, Hata A: Control of phenotypic plasticity of smooth muscle cells by bone morphogenetic protein signaling through the myocardin-related transcription factors. J Biol Chem 2007;282:37244-37255.

8 Li C, Xu Q: Mechanical stress-initiated signal transduction in vascular smooth muscle cells in vitro and in vivo. Cell Signal 2007;19:881-891.

9 Mayr M, Hu Y, Hainaut H, Xu Q: Mechanical stress-induced DNA damage and rac-p38MAPK signal pathways mediate p53-dependent apoptosis in vascular smooth muscle cells. FASEB J 2002;16:1423-1425.

10 Chen Z, Peng IC, Cui X, Li YS, Chien S, Shyy JY: Shear stress, SIRT1, and vascular homeostasis. Proc Natl Acad Sci U S A 2010;107:10268-10273.

11 Gao P, Xu TT, Lu J, Li L, Xu J, Hao DL, Chen HZ, Liu DP: Overexpression of SIRT1 in vascular smooth muscle cells attenuates angiotensin II-induced vascular remodeling and hypertension in mice. J Mol Med (Berl) 2014;92:347-357.

12 Guarani V, Potente M: SIRT1 - a metabolic sensor that controls blood vessel growth. Curr Opin Pharmacol 2010;10:139-145.

13 Archer SL: Pre-B-cell colony-enhancing factor regulates vascular smooth muscle maturation through a NAD+-dependent mechanism: recognition of a new mechanism for cell diversity and redox regulation of vascular tone and remodeling. Circ Res 2005;97:4-7.

14 Hsu CP, Zhai P, Yamamoto T, Maejima Y, Matsushima S, Hariharan N, Shao D, Takagi H, Oka S, Sadoshima J: Silent information regulator 1 protects the heart from ischemia/reperfusion. Circulation 2010;122:21702182.

15 Rizki G, Iwata TN, Li J, Riedel CG, Picard CL, Jan M, Murphy CT, Lee SS: The evolutionarily conserved longevity determinants HCF-1 and SIR-2.1/SIRT1 collaborate to regulate DAF-16/FOXO. PLoS Genet 2011;7:e1002235.

16 Yang X, Gong Y, Tang Y, Li H, He Q, Gower L, Liaw L, Friesel RE: Spry1 and Spry4 differentially regulate human aortic smooth muscle cell phenotype via Akt/FoxO/myocardin signaling. PloS one 2013;8:e58746.

17 Tucka J, Yu H, Gray K, Figg N, Maguire J, Lam B, Bennett M, Littlewood T: Akt1 regulates vascular smooth muscle cell apoptosis through FoxO3a and Apaf1 and protects against arterial remodeling and atherosclerosis. Arterioscler Thromb Vasc Biol 2014;34:2421-2428.

18 Ding M, Carrao AC, Wagner RJ, Xie Y, Jin Y, Rzucidlo EM, Yu J, Li W, Tellides G, Hwa J, Aprahamian TR, Martin KA: Vascular smooth muscle cell-derived adiponectin: a paracrine regulator of contractile phenotype. J Mol Cell Cardiol 2012;52:474-484.

19 McBurney MW, Yang X, Jardine K, Hixon M, Boekelheide K, Webb JR, Lansdorp PM, Lemieux M: The mammalian SIR2alpha protein has a role in embryogenesis and gametogenesis. Mol Cell Biol 2003;23:3854.

20 Yao H, Chung S, Hwang JW, Rajendrasozhan S, Sundar IK, Dean DA, McBurney MW, Guarente L, Gu W, Ronty M, Kinnula VL, Rahman I: SIRT1 protects against emphysema via FOXO3-mediated reduction of premature senescence in mice. J Clin Invest 2012;122:2032-2045.

21 Yao QP, Zhang P, Qi YX, Chen SG, Shen BR, Han Y, Yan ZQ, Jiang ZL: The role of SIRT6 in the differentiation of vascular smooth muscle cells in response to cyclic strain. Int J Biochem Cell Biol 2014;49:98-104.

22 Yao QP, Qi YX, Zhang P, Cheng BB, Yan ZQ, Jiang ZL: SIRT1 and Connexin40 Mediate the normal shear stressinduced inhibition of the proliferation of endothelial cells co-cultured with vascular smooth muscle cells. Cell Physiol Biochem 2013;31:389-399.

23 Reusch P, Wagdy H, Reusch R, Wilson E, Ives HE: Mechanical strain increases smooth muscle and decreases nonmuscle myosin expression in rat vascular smooth muscle cells. Circ Res 1996;79:1046-1053.

24 Williams B: Mechanical influences on vascular smooth muscle cell function. J Hypertens. 1998;16:19211929. 


\section{Cellular Physiology Cell Physiol Biochem 2015;37:1817-1829 \begin{tabular}{l|l|l} 
DOI: 10.1159/000438544 & (C) 2015 S. Karger AG, Basel
\end{tabular} www.karger.com/cpb \\ Huang et al.: SIRT1 Modulates Stretch Induced VSMC Differentiation}

25 Kim S, Hata A, Kang H: Down-regulation of miR-96 by bone morphogenetic protein signaling is critical for vascular smooth muscle cell phenotype modulation. J Cell Biochem 2014;115:889-895.

26 Yao Q, Hayman DM, Dai Q, Lindsey ML, Han HC: Alterations of pulse pressure stimulate arterial wall matrix remodeling. J Biomech Eng 2009;131:101011.

27 Chapman GB, Durante W, Hellums JD, Schafer AI: Physiological cyclic stretch causes cell cycle arrest in cultured vascular smooth muscle cells. Am J Physiol Heart Circ Physiol 2000;278,H748-754.

28 Zeidan A, Nordstrom I, Dreja K, Malmqvist U, Hellstrand P: Stretch-dependent modulation of contractility and growth in smooth muscle of rat portal vein. Circ Res 2000;87:228-234.

29 Simic P, Zainabadi K, Bell E, Sykes DB, Saez B, Lotinun S, Baron R, Scadden D, Schipani E, Guarente L: SIRT1 regulates differentiation of mesenchymal stem cells by deacetylating beta-catenin. EMBO Mol Med 2013;5:430-440.

30 Xiong S, Salazar G, Patrushev N, Ma M, Forouzandeh F, Hilenski L, Alexander RW: Peroxisome proliferatoractivated receptor gamma coactivator-1 alpha is a central negative regulator of vascular senescence. Arterioscler Thromb Vasc Biol 2013;33:988-998.

31 North BJ, Verdin E: Sirtuins: Sir2-related NAD-dependent protein deacetylases. Genome Biol 2004;5:224.

32 Orimo M, Minamino T, Miyauchi H, Tateno K, Okada S, Moriya J, Komuro I: Protective role of SIRT1 in diabetic vascular dysfunction. Arterioscler Thromb Vasc Biol 2009;29,889-894.

33 Csiszar A, Labinskyy N, Jimenez R, Pinto JT, Ballabh P, Losonczy G, Pearson KJ, de Cabo R, Ungvari Z: Antioxidative and anti-inflammatory vasoprotective effects of caloric restriction in aging: role of circulating factors and SIRT1. Mech Ageing Dev 2009;130:518-527.

34 Potente M, Dimmeler S. 2008. Emerging roles of SIRT1 in vascular endothelial homeostasis. Cell Cycle 2006; 7:2117-2122.

35 Alcendor RR, Gao S, Zhai P, Zablocki D, Holle E, Yu X, Tian B, Wagner T, Vatner SF, Sadoshima J: Sirt1 regulates aging and resistance to oxidative stress in the heart. Circ Res. 2007;100:1512-1521.

36 Gorenne I, Kumar S, Gray K, Figg N, Yu H, Mercer J, Bennett M: Vascular smooth muscle cell sirtuin 1 protects against DNA damage and inhibits atherosclerosis. Circulation 2013;127:386-396.

37 Kim HJ, Joe Y, Yu JK, Chen Y, Jeong SO, Mani N, Cho GJ, Pae HO, Ryter SW, Chung HT. Carbon monoxide protects against hepatic ischemia/reperfusion injury by modulating the miR-34a/SIRT1 pathway. Biochim Biophys Acta 2015;1852:1550-1559.

38 Cheng BB, Qu MJ, Wu LL, Shen Y, Yan ZQ Zhang P, Qi YX, Jiang ZL. MicroRNA-34a targets Forkhead box j2 to modulate differentiation of endothelial progenitor cells in response to shear stress. J Mol Cell Cardiol 2014;74:4-12.

39 Cheng BB, Yan ZQ, Yao QP, Shen BR, Wang JY, Gao LZ, Li YQ, Yuan HT, Qi YX, Jiang ZL: Association of SIRT1 expression with shear stress induced endothelial progenitor cell differentiation. J Cell Biochem 2012;113:3663-3671.

40 Giannakou ME, Partridge L: The interaction between FOXO and SIRT1: tipping the balance towards survival. Trends Cell Biol 2004;14:408-412.

41 Brunet A, Sweeney LB, Sturgill JF, Chua KF, Greer PL, Lin Y, Tran H, Ross SE, Mostoslavsky R, Cohen HY, Hu LS, Cheng HL, Jedrychowski MP, Gygi SP, Sinclair DA, Alt FW, Greenberg ME: Stress-dependent regulation of FOXO transcription factors by the SIRT1 deacetylase. Science 2004;303:2011-2015.

42 Li H, Liang J, Castrillon DH, DePinho RA, Olson EN, Liu ZP: FoxO4 regulates tumor necrosis factor alphadirected smooth muscle cell migration by activating matrix metalloproteinase 9 gene transcription. Mol Cell Biol 2007;27:2676-2686.

43 Paik JH: FOXOs in the maintenance of vascular homoeostasis. Biochem Soc Trans 2006;34:731-734.

44 Lee HY, Chung JW, Youn SW, Kim JY, Park KW, Koo BK, Oh BH, Park YB, Chaqour B, Walsh K, Kim HS: Forkhead transcription factor FOXO3a is a negative regulator of angiogenic immediate early gene CYR61, leading to inhibition of vascular smooth muscle cell proliferation and neointimal hyperplasia. Circ Res 2007;100:372-380.

45 Liu ZP, Wang Z, Yanagisawa H, Olson EN: Phenotypic modulation of smooth muscle cells through interaction of Foxo4 and myocardin. Dev Cell 2005;9:261-270. 